\title{
Frequency Domain Q Analysis and Stabilized Inverse Q Filtering: Blake Ridge data application
}

\author{
Edmarley Costa Ramos ${ }^{1}$, Felipe Timóteo da Costa ${ }^{1}$, Marco Antonio Cetale Santos ${ }^{1}$, Luiz Alberto Santos ${ }^{12}$, Rogério de \\ Araujo Santos ${ }^{12}$, Djalma Manuel Soares Filho ${ }^{2}$ \\ ${ }^{1}$ GISIS-UFF \\ ${ }^{2}$ Petrobras
}

Copyright 2019, SBGf - Sociedade Brasileira de Geofísica.

This paper was prepared for presentation at the $16^{\text {th }}$ International Congress of the Brazilian Geophysical Society, held in Rio de Janeiro, Brazil, August 19-22, 2019.

Contents of this paper were reviewed by the Technical Committee of the $16^{\text {th }}$ International Congress of The Brazilian Geophysical Society and do not necessarily represent any position of the SBGf, its officers or members. Electronic reproduction or storage of any part of this paper for commercial purposes without the written consent of The Brazilian Geophysical Society is prohibited.

\section{Abstract}

Seismic absorption can compromise seismic data quality by introducing phase distortion and loss of frequency content, specially high frequencies. Post stack constant $Q$ estimation was caried out by using the frequency peak shift method. Frequency domain $Q$ estimation methods are considered more reliable and robust. Stabilized inverse $Q$ filtering was applied to the data, recovering the attenuated frequencies and minimizing phase distortion. The filtered data displays increased resolution and a flatter amplitude spectrum ,while maintaning the signal to noise ratio.

\section{Introduction}

The Seismic absorption subject is no novelty, nevertheless it remains quite relevant up to this day given the constant need to improve seismic imaging in increasingly complex areas.

In the exploration seismology context, as the injected energy travels through the earth, an ineslastic medium, the wavefield is constantly deformed under the effects of attenuation and dispersion (non stationary behaviour) (Yilmaz, 2001). Different frequencies will travel with different phase velocities indulcing a phase distortion and the loss of frequency content, specially the higher frequencies, cause a pulse-broadening effect accompanied by an amplitude reduction.

Left untreated, seismic data quality may suffer from mispositioning of events, defocusing, amplitude and resolution loss (fig. 1), directly imparing seismic interpretation and quantitative analysis (e.g. inversion, AVO etc.).

Seismic Inverse $Q$ Filtering (IQF) technique, also known as deabsorption filtering, seeks to correct phase delays and recover the attenuated bandwidth but low signal to noise ratio (SRN) and reliable Quality Factor estimation are examples of challanges faced by geoscientists.

In this work, the core of $Q$ analysis and IQF are discussed; a constant quality factor value is estimated from stacked data using the Frequency Peak Shift (FPS) method and
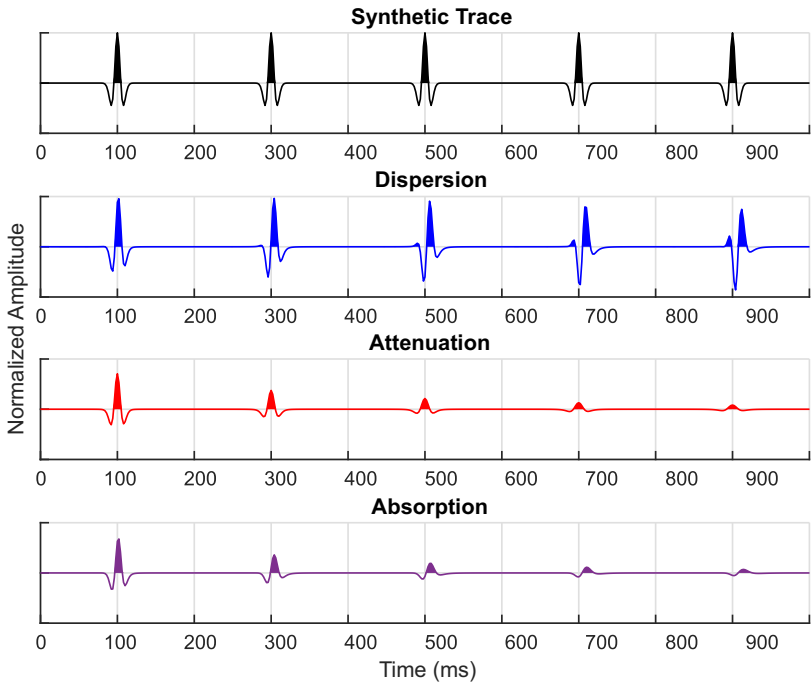

Figure 1: Synthetic seismic trace generated using a simetrical $50 \mathrm{~Hz}$ ricker wavelet at every $200 \mathrm{~ms}$. Absorption effects can be seen separately for a $Q$ value of 50 . Dispersion introduces phase distortion, mispositioning events and defocusing the data. Attenuation is responsible for a severe loss of amplitude and vertical resolution.

Stabilized Inverse $Q$ Filtering (SIQF) (Wang, 2008) is applied to the 3D Blake Ridge seismic dataset.

\section{Materials and Methods}

In this work an introduction of the basic theory of seismic absorption and inverse $Q$ fintering is done. Synthetic data was modelled to better illustrate key concepts. Frequency domain $Q$ estimation will be performed in the 3D Blake Ridge seismic data using the Frequency Peak Shift (FPS) method. Stabilized Inverse $Q$ Filtering (SIQF) is applied, seeking to recover the attenuated bandwidth and correct phase distortion.

\section{Sesmic Absorption and the Quality Factor}

The Quality Factor, central keypoint of seismic absorption, can be defined as a measurement of energy loss ratio per cicle in a anelastic system:

$$
Q=\left(\frac{1}{2 \pi} \frac{\Delta W}{W}\right)^{-1},
$$

Note that $Q$ is dimensionless and it is simply a way to 
express energy loss, not providing any information about the physical mechanism responsible for the dissipation.

Born (1941) proposes two possible main attenuation mechanisms: viscous attenuation and solid friction. In losses by viscous attenuation, the amount of lost energy per cicle is proportional to the ciclic stress frequency. In solid friction alternatively, the loss depends only on the nature of the material and the maximum amplitude strain, regardless of the frequency.

Usual marine seismic data possesses an overall low frequency content, therefore, the Quality Factor is assumed to be constant over this frequency range, suggesting energy loss by solid friction (Born, 1941; Liner, 2012).

Fluid saturation can also strongly influence absorptions behaviour. Born (1941); Winkler and Nur (1982) indicate that an initially constant $Q$ can present frequency variability due to saturation, therefore, viscous attenuation can play an important role in shallow and unconsolidated sediments.

Even without knowledge of the working mechanisms behind attenuation, its behaviour is well-known: Born (1941) establishes that the amplitude decrement with the travelled distance must be of an exponential form:

$$
A_{x}=A_{0} \exp ^{-\alpha(\omega) x}
$$

The decrement is controled by the variable $\alpha$, known as attenuation coefficient, defined by the medium properties and as a function of $Q$ and frequency, it dictates attenuation individually for each frequency:

$$
\alpha(\omega)=\frac{\omega}{2 c Q},
$$

where $c$ is the group velocity.

Consider the following 1-D one-way wave equation equation and its analytical solution (Wang, 2008):

$$
\begin{gathered}
\frac{\partial U(x, \omega)}{\partial x}=-i k(\omega) U(x, \omega), \\
U(x+\Delta x, \omega)=U(x, \omega) \exp [-i k(\omega) \Delta x],
\end{gathered}
$$

where $U(x, \omega)$ is a plane wave with angular frequency $\omega$ after travelling a distance $\Delta x, k(\omega)$ is the wavenumber and $i$, the imaginary unit. To introduce the effects of absorption, the wavenumber $k$ must assume a complex value that includes both the attenuation coefficient and the dispersive wavenumber $(\kappa)$ :

$$
k(\omega)=\kappa(\omega)-i \alpha(\omega)
$$

eq. 5 then takes the form

$$
U(x+\Delta x, \omega)=U(x, \omega) \exp [-\alpha(\omega) \Delta x] \exp [-i \kappa(\omega) \Delta x] .
$$

Equation 7 express two clearly defined terms: the amplitude and phase terms, responsible each for attenuation and dispersion of the wave during propagation, respectivelly.

Futterman (1962) suggests that the relationship between the attenuation-dispersion pair must be of a KramersKronig (KK) type, where the real part of $k$ can be determined from the sum of the imaginary part over the entire frequency bandwidth (Wang, 2008; Liner, 2012):

$$
\operatorname{Re}(k)=\frac{\omega}{v(\omega)} \equiv \kappa \approx \mathscr{H}\{\alpha(\omega)\},
$$

where $\mathscr{H}\{\cdot\}$ denotes the Hilbert transform. This implies the causality of the signal. Amplitude attenuation must be accompanied by phase delays as the energy travels the earth in order to maintain the causality principle. That is, broadly speaking, there is no attenuation without dispersion.

Different mathematical $Q$ models are characterised by their own definition of the complex wavenumber. The Kolsky $Q$ model (Kolsky, 1963) is widely used for Inverse $Q$ Filter design due to its flexibility and simple parameterization (Wang, 2008).

Introducing a variable change $\Delta \tau=\Delta x / v$ and using Kolsky's model, equation 7 can be written as:

$$
\begin{aligned}
U(\tau+\Delta \tau, \omega)= & U(\tau, \omega) \exp \left[-\frac{\pi f \Delta \tau}{Q}\right] \\
& \times \exp \left[-\frac{i \omega \Delta \tau}{\pi Q} \ln \left(\frac{\omega}{\omega_{r}}\right)\right],
\end{aligned}
$$

where $\Delta \tau$ is the time step and $\omega_{r}$ the angular reference frequency. Equation 9 represents the frequency domain wave propagation through absorptive media (fig. 2) and is the basis for designing the inverse $Q$ filter employed in this work.

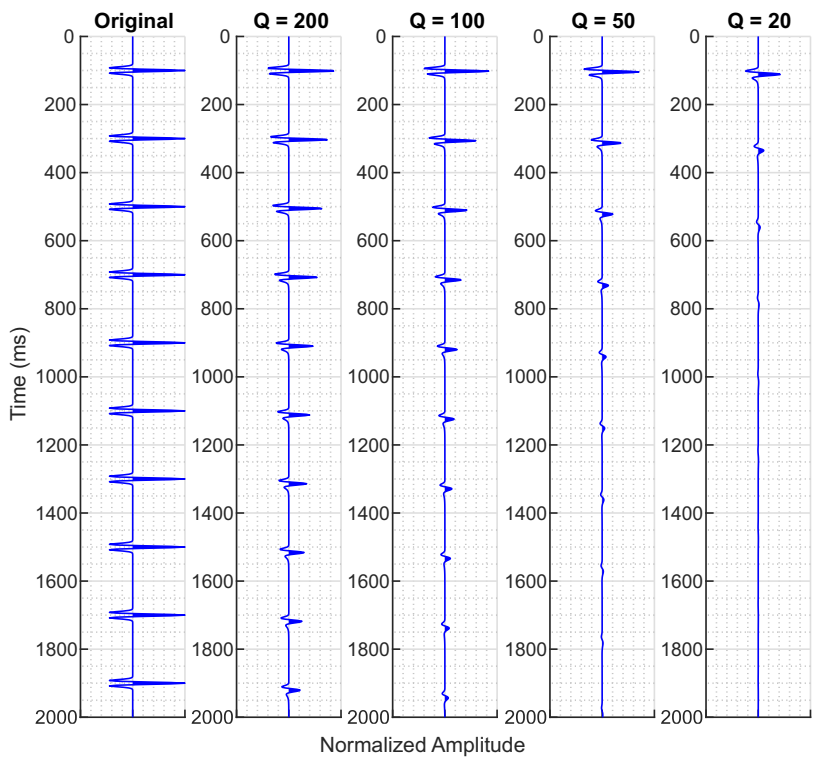

Figure 2: Synthetic data modelled with absorption for different $Q$ values.

This approach of computing the wavefield by sucessive 
time steps in frequency domain is described by Wang (2008) and refered as the downward continuation approach. The time-domain signal is given by applying the imaging condition, as in seismic migration, that is, by the sum of individual downward continued plane waves:

$$
u(\tau+\Delta \tau)=\frac{1}{\pi} \int_{0}^{\infty} U(\tau+\Delta \tau, \omega) d \omega
$$

\section{Inverse $Q$ Filtering and Stability}

Equation 9 describes the earth's $Q$ filter. Synthetic data was modelled using consecutives ricker wavelets for different $Q$ values to simulate earth's absorption (fig. 2). Considering the amplitude and phase terms (eq. 9) as

$$
\Gamma(\tau+\Delta \tau)=\exp \left[-\frac{\pi f \Delta \tau}{Q}\right]
$$

and

$$
\Phi(\tau+\Delta \tau)=\exp \left[-\frac{i \omega \Delta \tau}{\pi Q} \ln \left(\frac{\omega}{\omega_{r}}\right)\right]
$$

respectivelly, a simple inverse $Q$ filter can be then written by simply inverting the signal of the exponential functions (Ribeiro et al., 2015)

$$
U(\tau+\Delta \tau, \omega)=U(\tau, \omega) \frac{1}{\Gamma(\tau+\Delta \tau)} \frac{1}{\Phi(\tau+\Delta \tau)}
$$

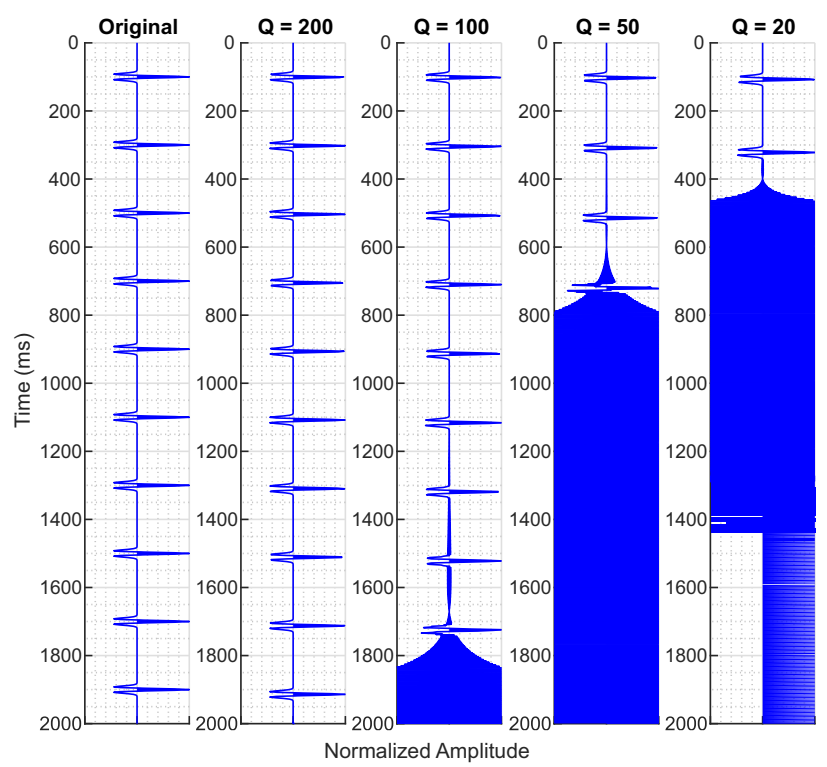

Figure 3: Synthetic data after basic inverse $Q$ filtering (eq. 13), no stabilization strategy was applied.

Figure 3 shows the result of applying eq. 13 directly to the modelled data using the correct $Q$ values. For $Q=200$ the amplitude is recovered and phase corrected, but for the other values, strong artifacts can be seen, even if the synthetics are noise free, specially on lower $Q$ and higher time values.
These artifacts are a natural consequence of the downward continuation procedure, referred as the inverse $Q$ filtering numerical instability. The downward continuated plane waves are constantly attenuated, at some point their magnitude inexpressively small, lower or at the same level as the ambient noise level. In this specific example (noisefree), the amplified noise com from numerical machine errors in the working precision.

Maximum gain limits and bandpass filtering are used to overcome instability and suppress excessive noise amplification, alternatively, the phase correction is inherently stable.

This work adopts the stabilization strategy of Wang (2008), that employs a modified version of Kolsky's $Q$ model to respect the Kramers-Kronig relationship, ensures causality (Futterman, 1962; Wang, 2008), and defines a stabilized inverse $Q$ filtering amplitude operator.

Considering the initial time step $\tau_{0}=0$, Wang (2008) defines the Stabilized Inverse $Q$ Filtering as

$$
\begin{aligned}
u(\tau)= & \frac{1}{\pi} \int_{0}^{\infty} U(0, \omega) \Lambda(\tau, \omega) \\
& \times \exp \left[i \int_{0}^{\tau}\left(\frac{\omega}{\omega_{h}}\right)^{-\gamma\left(\tau^{\prime}\right)} \omega d \tau^{\prime}\right] d \omega,
\end{aligned}
$$

where

$$
\begin{gathered}
\gamma(\tau)=\frac{1}{\pi Q(\tau)}, \\
\beta(\tau, \omega)=\exp \left[-\int_{0}^{\tau} \frac{\omega}{2 Q\left(\tau^{\prime}\right)}\left(\frac{\omega}{\omega_{h}}\right)^{-\gamma\left(\tau^{\prime}\right)} d \tau^{\prime}\right], \\
\Lambda(\tau, \omega)=\frac{\beta(\tau, \omega)+\sigma^{2}}{\beta^{2}(\tau, \omega)+\sigma^{2}} .
\end{gathered}
$$

$\beta(\tau, \omega)$ is the $Q$ filter amplitude operator; $\Lambda(\tau, \omega)$ the stabilized inverse $Q$ filter amplitude operator and $\sigma^{2}$ is the stabilization factor.

The Stabilized Amplitude Operator (fig. 4) enables the effective recovery of the signal bandwidth by the use of a dynamic gain, function of $Q$, travel time and frequency, leaving undesirable frequency components untouched. The IQF is then stabilized (fig. 5) the SNR, preserved.

Frequency Domain $Q$ Estimation: Frequency Peak Shift Method

The Frequency Peak Shift (FPS) Method, first described by Quan and Harris (1997), derives a $Q$ value based on the displacement of the measured frequency peak (FP) between 2 time windows. The suffered attenuation is directly proportional to the frequency peak shift towards the lower frequencies (fig. 6).

Zhang and Ulrych (2002) adopted a Ricker Wavelet to represent the data amplitude spectrum:

$$
w(f)=\frac{2}{\sqrt{\pi}} \frac{f^{2}}{f_{p 0}^{2}} \exp \left(\frac{f^{2}}{f_{p 0}^{2}}\right),
$$




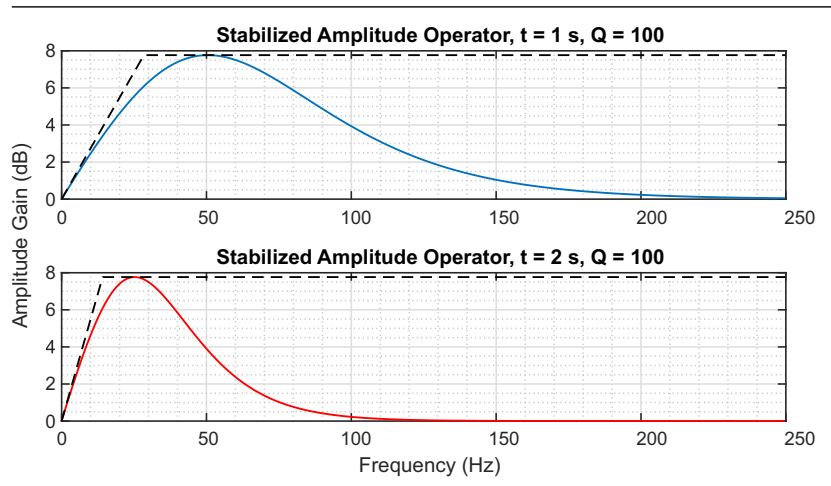

Figure 4: Stabilized Amplitude Operator $\Lambda(\tau, \omega)$ (eq. 17) for $Q=100$ at 1 and 2 seconds. Note that at 2 seconds, the gain at frequencies higher than $100 \mathrm{~Hz}$ is almost insignificant, this can be interpreted as, given the $Q$ value employed, the signal would lack these higher frequencies at this time, beyond recovering, so the algorithm selects the effective recovey range base on the travel time, frequency and $Q$. The Gain limit stabilization approach is represented by the dashed black line.

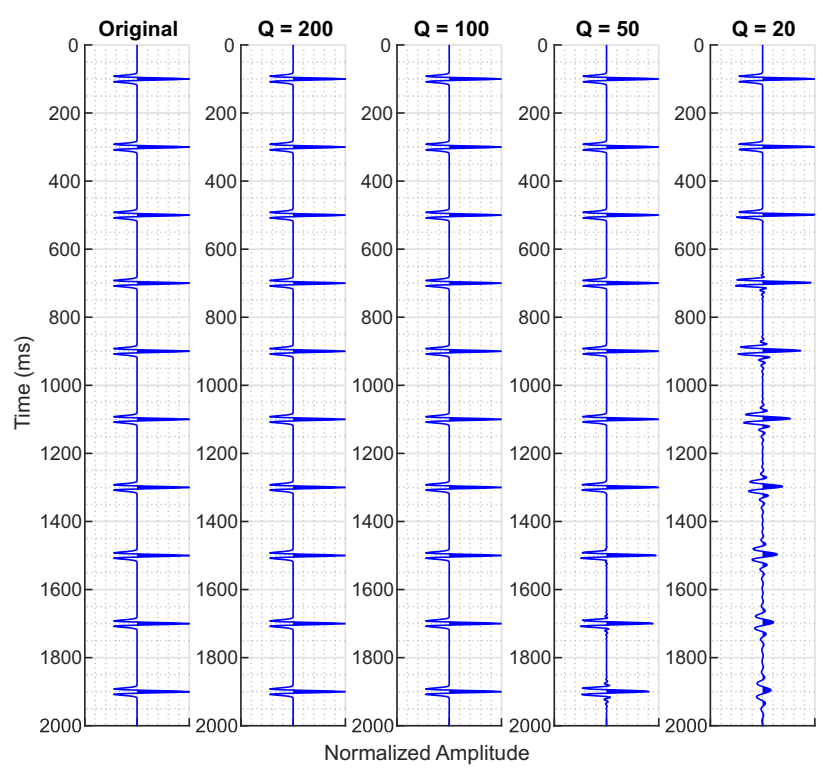

Figure 5: Synthetic data after Stabilized Inverse $Q$ Filtering.

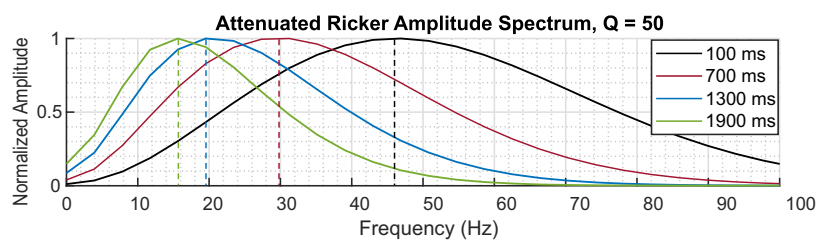

Figure 6: Attenuated Ricker Wavelet Spectra for $Q=50$. The displacement of the frequency peak torwards the low frequencies is the basis of the FPS $Q$ estimation method.

where $f_{p 0}$ is the ricker peak frequency. The wavelet amplitude after propagation on a anelastic media will be given by a combination of eq. 18 and the amplitude term from eq. 9:

$$
w(f, t)=w(f, 0) e^{-\frac{\pi f t}{Q}} .
$$

The frequency peak can be then derived at each point:

$$
\frac{\partial w(f, t)}{\partial f}=0
$$

Ultimately, effective $Q$ values can be calculated from the picked FP and a referece frequency peak at a given reference time:

$$
Q_{e f f}=\frac{\pi t f_{p} f_{p r e f}^{2}}{2\left(f_{p r e f}^{2}-f_{p}^{2}\right)},
$$

where $t$ is the traveled time, $f_{p}$ is the measured attenuated frequency peak and $f_{\text {pref }}$ is the reference frequency peak.

\section{Results}

$Q$ analysis was performed on the 3D Blake Ridge seismic data (fig. 7), that exhibit expressive absortion due to a complex methane hidrate and free gas system (Holbrook et al., 2002; Hornbach et al., 2008).

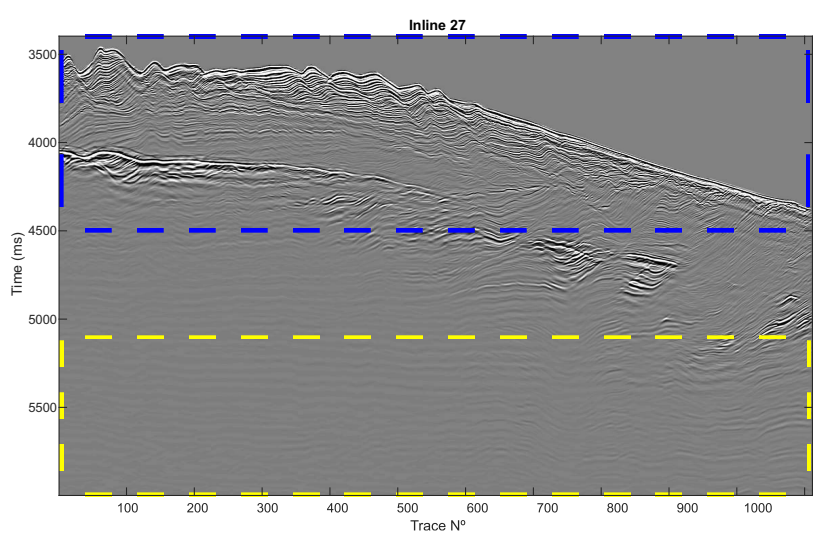

Figure 7: Inline 27 of the 3D Blake Ridge seismic data (Hornbach et al., 2008). The shallow and deep windows used to estimate the $Q$ factor are represented by dashed lines of the corresponding color.

Two windows, in the shallow and deep parts, were used for $Q$ estimation using the FPS method. Figure 8 displays the normalized amplitude spectrum for these 2 windows. The displacement of the FP from $56.88 \mathrm{~Hz}$ to $22.21 \mathrm{~Hz}$ in less than 2 seconds suggest high absorption anomalies related to gas saturation.

A $Q$ value of 74.12 was derived by using equation 21 . This constant value represents the general absorption behavior for this line and it is coherent to the expected given the geological background of the region.

SIQF was applied utilizing the newfound $Q$ and a stabilization factor $\sigma^{2}=1 \%$. The results can be compared in the figures 9 and 10, where a deeper portion of the section can be seen. At higher times the absortion effect is more pronounced and the benefits of the SIQF can be better appraised. 


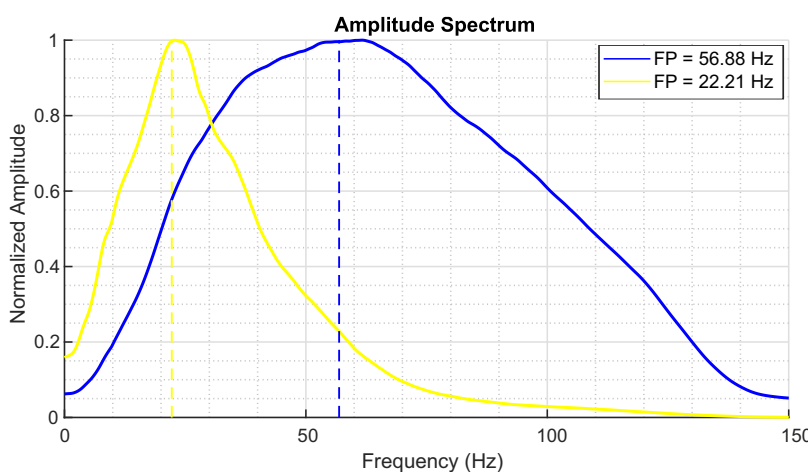

Figure 8: Normalized Amplitude Spectrum of the shallow and deep windows. Strong seismic absorption due to free gas and hydrate areas caused the FP to substantially decrease in a short time frame.

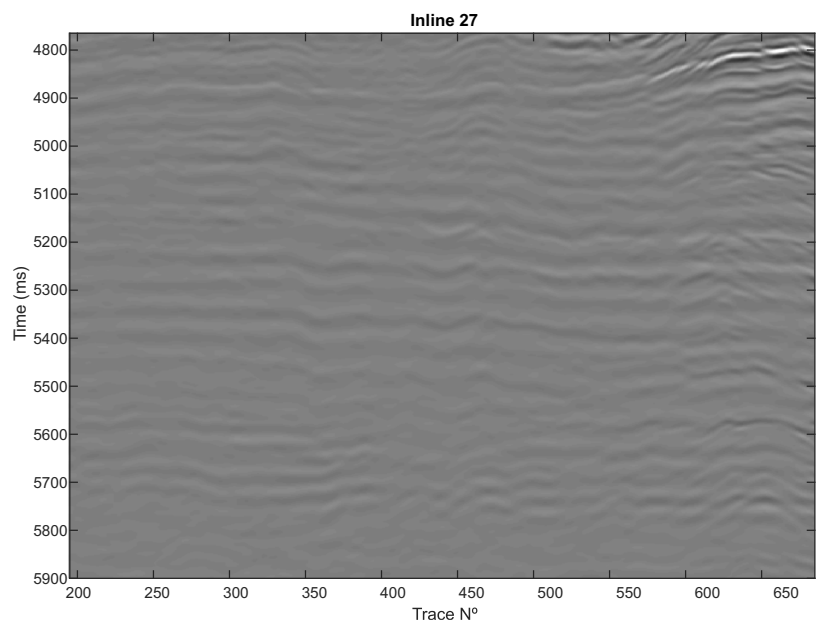

Figure 9: Deep portion of inline 27 of the 3D Blake Ridge seismic data - before SIQF.

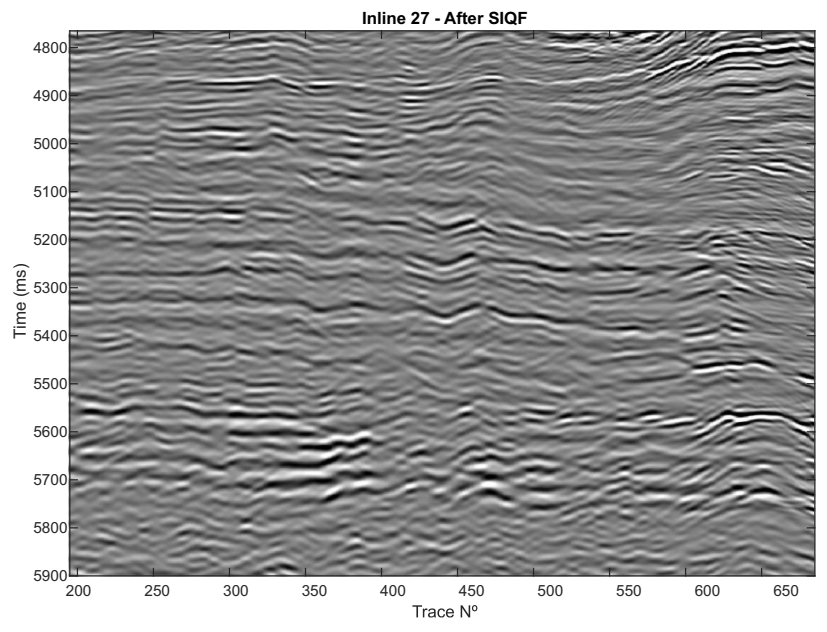

Figure 10: Deep portion of inline 27 of the 3D Blake Ridge seismic data - after SIQF. Structures can be clearly observed as the data enjoys a higher temporal resolution and focusing after phase distortion correction and amplitude recovery.
The filtered data displays a higher level of detail as a result of the phase distortion correction and the recovery of frequency content. The amplitude spectrum of the displayed deep portion of the data, before and after application of the SIQF, is shown in figure 11. It can be seen that the SIQF significantly recovered the attenuated frequencies, extending the bandwidth and increasing resolution while preserving the SNR.

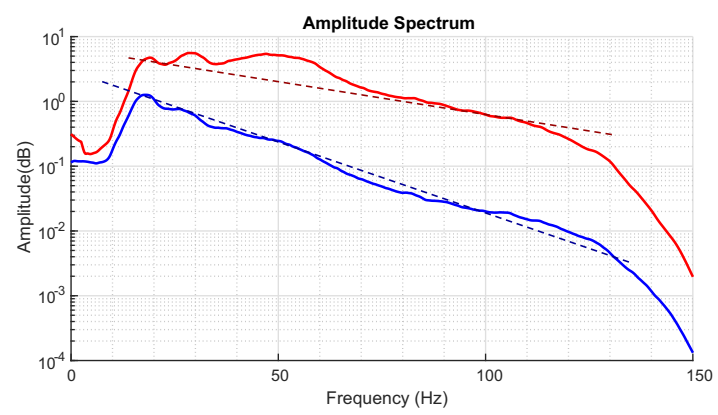

Figure 11: Amplitude Spectrum of the deeper segment of inline 27 of the Blake Ridge 3D seismic data. The SIQF recovered part of the attenuated high frequencies, extending the bandwidth and enhancing resolution while preserving the SNR. The filtered spectrum is overall flatter.

\section{Discussion and Conclusion}

Quality Factor estimation still poses a challenge for many geoscientists even to this day. Several works enjoyed relative success by the use of Vertical Seismic Profile data. Unfortunately, such data is hardly available and the information is localized at best.

Laboratory measurements granted important insights about the anelastic behaviour, they have shown that absorption is controlled by a plethora of parameters in a very complex manner, such as material properties (matrix and fluid); saturation; pressure; temperature; etc. (Born, 1941; Winkler and Nur, 1982; Dasgupta and Clark, 1998).

Though for seismic data processing purposes, $Q$ laboratory estimatives are not reliable due to the frequency gap between the employed tools (capable of reaching $10^{3}-$ $10^{6} \mathrm{~Hz}$ ) and actual seismic data.

Data driven $Q$ estimation methods became increasingly popular, providing processors with suitable results and enabling high spatial coverage, trend followed by the industry over the recent past (e.g. Dasgupta and A. Clark, 1994; Dasgupta and Clark, 1998; Taner and Treitel, 2003; Cavalca et al., 2011; Raji and Rietbrock, 2013; Liu and Zhang, 2017).

Even so, reliable $Q$ estimation proves to be challenging. Decoupling intrinsic attenuation effects from wave propagation one's (apparent attenuation) and low signal to noise ratio are examples. Frequency domain methods for $Q$ estimation became increasingly popular, as they allow for more robustness and disconsiders frequency independent phenomena.

The frequency peak shift method was applied to the 3D Blake Ridger seismic data, the resulting $Q$ is coherent with the geological background of the area. Stabilized Inverse $Q$ Filtering was employed, the attenuated frequency 
range was substantially recovered and phase distortions minimized. The data displays a flatter spectrum and the signal to noise ratio was conserved.

\section{References}

Born, W. T., apr 1941, The Attenuation Constant of Earth Materials: GEOPHYSICS, 6, no. 2, 132-148.

Cavalca, M., Moore, I., Zhang, L., Ng, S. L., Fletcher, R., and Bayly, M., jan 2011, Ray-based tomography for $Q$ estimation and $Q$ compensation in complex media: Raybased tomography for $Q$ estimation and $Q$ compensation in complex media:, Society of Exploration Geophysicists, SEG Technical Program Expanded Abstracts 2011, 3989-3993.

Dasgupta, R., and A. Clark, R., jun 1994, Successful estimation of $Q$ from surface seismic data - A case study: Successful estimation of $Q$ from surface seismic data A case study:, 56th Mtg., Session:H016.

Dasgupta, R., and Clark, R. A., nov 1998, Estimation of Q from surface seismic reflection data: GEOPHYSICS, 63, no. $6,2120-2128$.

Futterman, W. I., dec 1962, Dispersive Body Waves: Journal of Geophysical Research, 67, no. 13, 52795291.

Holbrook, W., Gorman, A., Hornbach, M., Hackwith, K., Nealon, J., Lizarralde, D., and Pecher, I., 2002, Seismic detection of marine methane hydrate: The Leading Edge, 21, no. 7, 686-689.

Hornbach, M. J., Saffer, D. M., Holbrook, W. S., Van Avendonk, H. J., and Gorman, A. R., 2008, Threedimensional seismic imaging of the blake ridge methane hydrate province: Evidence for large, concentrated zones of gas hydrate and morphologically driven advection: Journal of Geophysical Research: Solid Earth, 113, no. B7.

Kolsky, H., 1963, Stress waves in solids:, volume 1098 Courier Corporation.

Liner, C. L., jan 2012, Elements of Seismic Dispersion: A Somewhat Practical Guide to Frequency-Dependent Phenomena: Society of Exploration Geophysicists.

Liu, N., and Zhang, B., aug 2017, Q estimation using stretch compensated prestack gathers: $Q$ estimation using stretch compensated prestack gathers:, Society of Exploration Geophysicists, SEG Technical Program Expanded Abstracts 2017, 3505-3510.

Quan, Y., and Harris, J. M., 1997, Seismic attenuation tomography using the frequency shift method: Geophysics, 62, no. 3, 895-905.

Raji, W. O., and Rietbrock, A., sep 2013, Determination of quality factor (Q) in reflection seismic data: Determination of quality factor $(Q)$ in reflection seismic data:, Society of Exploration Geophysicists, SEG Technical Program Expanded Abstracts 2013, 31913195.
Ribeiro, K., Lewiner, T., and Duarte, O., aug 2015, On seismic absorption correction: On seismic absorption correction:, Brazilian Geophysical Society, 14th International Congress of the Brazilian Geophysical Society \& EXPOGEF, Rio de Janeiro, Brazil, 3-6 August 2015, 966-971.

Taner, M. T., and Treitel, S., jan 2003, A robust method for $Q$ estimation: A robust method for $Q$ estimation:, Society of Exploration Geophysicists, SEG Technical Program Expanded Abstracts 2003, 710-713.

Wang, Y., 2008, Seismic inverse Q filtering: Blackwell Pub.

Winkler, K. W., and Nur, A., jan 1982, Seismic Attenuation: Effects of Pore Fluids and Frictional-Sliding: GEOPHYSICS, 47, no. 1, 1-15.

Yilmaz, Ö., 2001, Seismic data analysis: Processing, inversion, and interpretation of seismic data: Society of exploration geophysicists.

Zhang, C., and Ulrych, T. J., 2002, Estimation of quality factors from cmp records: Geophysics, 67, no. 5, 15421547.

\section{Acknowledgments}

We would like to thank to GISIS Lab, for providing the necessary tools to perform this work; and OpendTect/dGB Earth Sciences for the seismic data. 